\title{
Tabu search algorithms for water network optimization
}

\author{
Maria da Conceição Cunha ${ }^{a, *}$, Luisa Ribeiro ${ }^{b}$ \\ a Dep. de Engenharia Civil, Universidade de Coimbra, PoloII-Pinhal de Marrocos, 3030-290 Coimbra, Portugal \\ ${ }^{\mathrm{b}}$ Instituto Superior de Engenharia de Coimbra, Quinta da Nora, 3031-601 Coimbra, Portugal
}

Received 15 March 2001; accepted 17 March 2003

Available online 12 August 2003

\begin{abstract}
In this paper we propose a tabu search algorithm to find the least-cost design of looped water distribution networks. The mathematical nature of this optimization problem, a nonlinear mixed integer problem, is at the origin of a multitude of contributions to the literature in the last 25 years. In fact, exact optimization methods have not been found for this type of problem, and, in the past, classical optimization methods, like linear and nonlinear programming, were tried at the cost of drastic simplifications. Tabu search is a valuable heuristic technique for solving problems cast in combinatorial form. This is based on the human memory process and uses an iterative neighborhood search procedure in an attempt to avoid becoming trapped in local optima. The use of such a heuristic procedure to solve the aforementioned problem needs particular tailoring to produce high quality solutions. In this paper we present the essential features of the algorithm and the results obtained when it is applied to some of the classical water distribution network case studies appearing in the literature. The results are very promising and demonstrate the usefulness of tabu search algorithms in solving this kind of optimization problem.
\end{abstract}

(c) 2003 Elsevier B.V. All rights reserved.

Keywords: Heuristics; Tabu search; Water distribution networks design; Optimization

\section{Introduction}

Water distribution networks are very important urban infrastructures whose realization needs huge investments. Therefore it is important that appropriate tools are used to help find the best decisions regarding their layout, design and operation. In the past twenty-five years a great deal of research has been conducted on the optimization of water distribution networks.

\footnotetext{
${ }^{*}$ Corresponding author.

E-mail addresses: mccunha@dec.uc.pt (M. da Conceição Cunha), luirenco@mail.isec.pt (L. Ribeiro).
}

In this paper we are going to deal with a looped distribution network design problem, which entails finding the set of commercial diameters (diameters are available only in certain sizes) that incur the least cost. The optimization model representing such a problem is a nonlinear mixed integer model. In fact, the equations representing the hydraulic behavior (equations coming from physics, representing flow and energy aspects) of the network, like the energy conservation law, are nonlinear, and the diameters must be chosen from the discrete diameters available commercially. These mathematical characteristics of the problem, which has discontinuous functions, with many 
local optima, have given rise to the multitude of methods proposed in the literature. In the past, methods like linear and nonlinear programming were used, sometimes at the cost of considerable simplifications of the optimization models. One of the earliest novel optimization approaches was presented by Alperovits and Shamir (1977). These authors proposed the so-called linear programming gradient method. This is an iterative method where, at each iteration, a fixed set of flows is tried, and every pipe is divided into segments, each with a different diameter. The decision variables are the lengths of each segment, and the problem is reduced to a linear one. In the successive iterations, the flow variables are heuristically adjusted according to the gradient of the objective function. Other authors followed this innovative course and introduced alternative derivations from the linear programming-based gradient expressions (Quindry et al., 1981; Fujiwara et al., 1987; Kessler and Shamir, 1989; Fujiwara and Khang, 1990). It should be noted that this approach leads to solutions in which pipes have one or two fixed diameter segments. This is called a split-pipe design, and solutions have been reported where $99 \%$ of a pipe has one diameter and the remaining $1 \%$ another. For practical implementation this type of solution is unrealistic. Another optimization approach uses nonlinear programming, in which the diameter is taken to be a continuous variable (Fujiwara and Khang, 1990, 1991; Varma et al., 1997). The solution provided by the continuous diameter approach is not practical, because diameters must be chosen from the diameter sets available commercially, and only certain sizes are marketed. The conversion of continuous solutions into discrete diameters can induce a loss of optimality and even a loss of feasibility. Readers interested in previous work regarding the split-pipe design and continuous diameter approaches are referred to Walski (1985) and Lansey and Mays (1989).

In the light of the above comments it is clear that diameters have to be considered as discrete variables. Gessler (1981, 1982, 1985) made some of the first attempts to treat diameters as discrete variables. This author uses an explicit enumeration, examining all possible diameter combina- tions. In order to overcome the dimensional difficulties encountered, similar classes of pipes were grouped together and assigned the same diameter. This is an interesting approach, but for large-scale problems, grouping in this way can induce a loss of information and render the method inefficient.

Methods capable of handling nonlinear mixed integer models (keeping all the realistic features), searching for global optimality, have only been developed recently. Among them we find genetic algorithms (used by Goldberg and Kuo, 1987; Hadji and Murphy, 1990; Murphy and Simpson, 1992; Walters and Cembrowicz, 1993; Simpson et al., 1994; Savic and Walters, 1997; Walters et al., 1999), and simulated annealing (used by Cunha and Sousa, 1999; Cunha and Sousa, 2001). Theoretical proofs relative to the convergence of simulated annealing with global optimal solutions have been formulated, but it would be impractical to observe theoretical conditions in real-world applications (Cunha and Sousa, 1999). Regarding genetic algorithms, only empirical results related to their convergence to global optimal solutions are known. The use of genetic and simulated annealing algorithms was a significant step forward in the search for the most suitable method for solving the least-cost design water distribution network problem. Cunha and Sousa $(1999,2001)$ showed that some improvements can be achieved in both solution quality and computer running times with simulated annealing vis-à-vis genetic algorithms. Nevertheless, it must still be pointed out that there is no proof that any of the solutions given by the optimization algorithms referred to above provide global optimality relative to the optimal design of water distribution networks. Research should continue, therefore, and the evaluation of other heuristic methods is still important. This is the main motivation for the present work. In this paper, the authors propose a tabu search algorithm for solving the aforementioned problem and compare the results with those achieved by genetic and simulated annealing algorithms. Tabu search is a heuristic procedure designed for solving optimization problems, and it has been successfully applied to a variety of combinatorial problems like scheduling, vehicle 
routing, traveling salesman problem, etc. Tabu search is a very aggressive heuristic for overcoming local optima, and searching for global optimality by exploring other regions of the solution space. Its efficiency depends on the fine-tuning of some parameters. Faigle and Kern (1992) presented convergence results for a probabilistic version of tabu search inspired by the analyses carried out for simulated annealing.

\section{Optimization model formulation}

The design problem can be stated as follows: to choose from a set of discrete commercial diameters, the combination of diameters that gives the least cost network required to supply a set of demand nodes within a prescribed range of pressures. The corresponding model (Cunha and Sousa, 1999) can be written as

$$
\begin{array}{ll}
\text { Min } & \sum_{k \in N P} c_{k}\left(D_{k}\right) L_{k} \\
\text { s.t. } & \sum_{i \in N P_{n}} \operatorname{sgn} Q_{i}=S_{n}, \quad \forall n \in N N, \\
& \Delta H_{k}=w \frac{L_{k}}{C_{k}^{\alpha} D_{k}^{\beta}} \operatorname{sgn}\left(Q_{k}\right)\left|Q_{k}\right|^{\alpha}, \quad \forall k \in N P, \\
& H_{n} \geqslant H_{n \text { min }}, \quad \forall n \in N N, \\
& D_{k} \geqslant D_{\min }, \quad \forall k \in N P, \\
& D_{k} \in\{D\}, \quad \forall k \in N P,
\end{array}
$$

where $N N$ : node set; $N P$ : pipe set; $N P_{n}$ : set of pipes entering or leaving node $n ;\{D\}$ : set of commercial diameters; $D_{k}$ : diameter of pipe $k ; L_{k}$ : length of pipe $k ; Q_{k}$ : flow in pipe $k ; S_{n}$ : demand at node $n ; \Delta H_{k}$ : head loss in pipe $k ; H_{n}$ : head in node $n ; H_{n \text { min }}$ : minimum head of node $n ; D_{\min }$ : minimum diameter; $c_{k}$ : unit cost per length; $w$ : numerical conversion constant; $C$ : roughness coefficient of Hazen-Williams equation (depending on the material); $\alpha, \beta$ : constants as proposed by Hazen-Williams.

The objective function (1) expresses the minimization of the network cost that is assumed to be a function of the pipe diameters (the decision variables), with the length $L_{k}$ and the unit $\operatorname{cost} c_{k}$ to be known. In fact, as the network layout is supposed to be established, the lengths are defined.
For each diameter $D_{k}$ of the diameter set, the unit cost is provided by commercial catalogues. This model assumes a gravity fed system not containing pumps and other devices.

The first set of constraints (2) expresses the mass conservation law at each node or the continuity principle.

The head loss in each pipe is the difference between its node heads (3). The Hazen-Williams equation is selected to represent these head losses.

The set of constraints (4) expresses the minimum pressure (technical requirements) at each node.

The set of constraints (5) expresses the acceptable minimum diameter requirements in each pipe.

The set of constraints (6) signifies that the diameter of each pipe must belong to a commercial set.

\section{Tabu search algorithms}

Tabu search is a global optimization heuristic based on the human memory process (for a detailed explanation of tabu search algorithms see Glover and Laguna (1997)). The implementation of this method is based on the exploration of the neighborhood of the current solution. This can be done by exploring either the entire neighborhood or a part of it, and so a mechanism for generating neighbor solutions must be provided. This mechanism defines the moves to be performed in each iteration. In order to prevent cycling between the same solutions, certain moves can be forbidden, earning them the status of "tabu move". The set of tabu moves defines the tabu list. Tabu moves are not permanent; a short-term memory function enables them to leave the tabu list. They stay in the tabu list for a set time (number of iterations) defined by the tabu tenure parameter. The use of the aspiration criterion permits certain moves on the tabu list to overcome any tabu status. This will occur whenever moves lead to the finding of solutions corresponding to a predefined criterion. A diversification procedure, using a long-term memory function, will lead to the exploration of regions of the solution space not previously visited. 


\section{Algorithm implementation and results}

The tabu search algorithm is implemented using some well-known test networks from the literature. The evaluation of different optimization techniques to solve least-cost design water network problems contained in the literature is based usually on three case studies: Alperovits and Shamir (Fig. 1), Hanoi (Fig. 2) and New York (Fig. 3) networks. Two more cases have been added here (Figs. 4 and 5), but only results from one or two researchers are available for comparison. In this field (least-cost design water network) there is no collection of benchmark of problems, as there is with some classical optimization problems (facility location, traveling salesman, etc.). It would be very useful to build up a "library" containing a significant number of case studies to permit the comparison of the results provide by different solution methods.

The data used here can be found in Cunha and Sousa (1999) for networks 1 and 2, in Cunha and Sousa (2001) for network 3, and in Sousa and Cunha (1999) for networks 4 and 5. Network 4 has been solved for two different minimum diameter constraints $\left(D_{\min }=2\right.$ and 4 in.). A single load condition (flow requirements) has been considered for all the networks except network 5, where three load conditions were used (one for supply and two others to satisfy fire safety requirements). In each iteration the algorithm uses demand supplies for sizing the network, but ensuring that fire safety

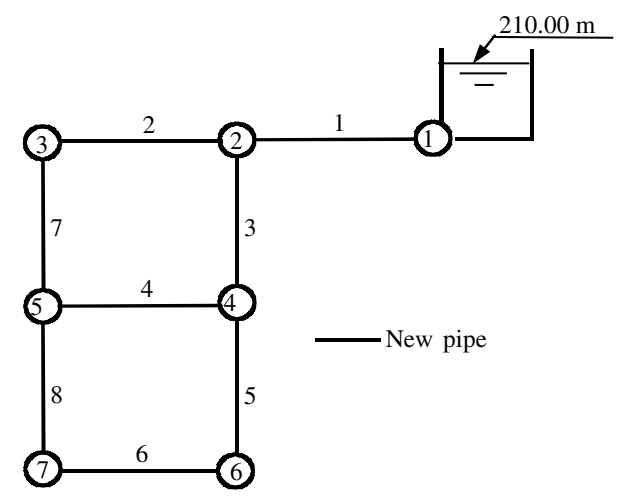

Fig. 1. Network 1 (Alperovits and Shamir, 1977).

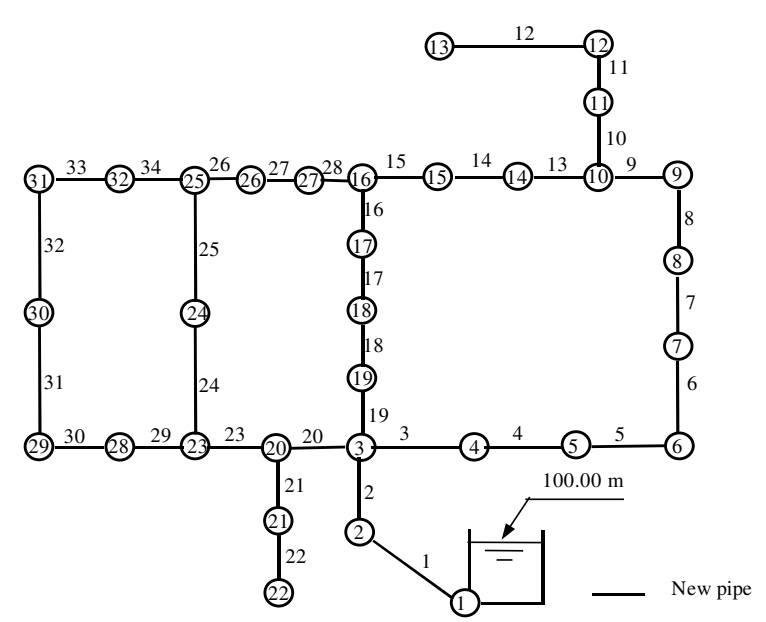

Fig. 2. Network 2 (Hanoi network).

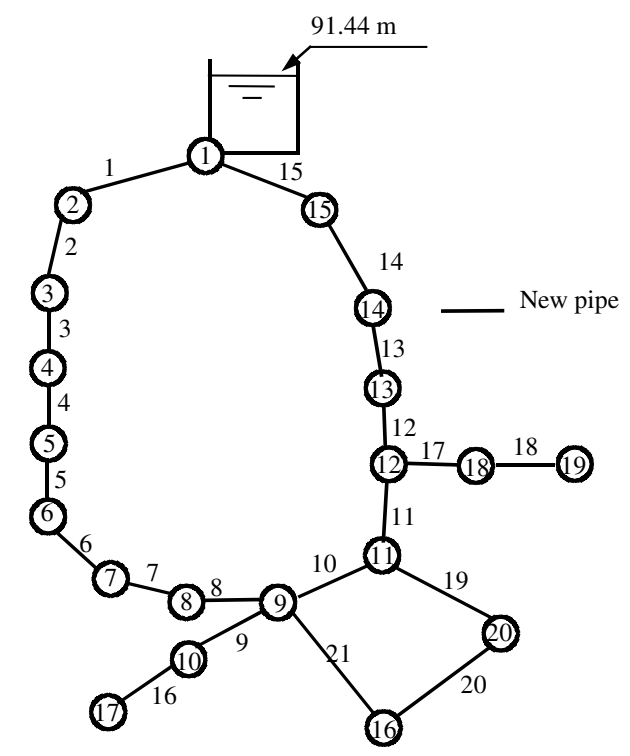

Fig. 3. Network 3 (New York network).

requirements are met. The design of network 5 involves building some new pipes and reinforcing some of the existing ones (1,4 and 5) by using pipes in parallel to increase network capacity.

The essential features that have been considered in building a tabu search algorithm for optimizing the design of a looped water distribution network are: the initial solution, the neighborhood structure, the diversification procedure, the tabu moves, 


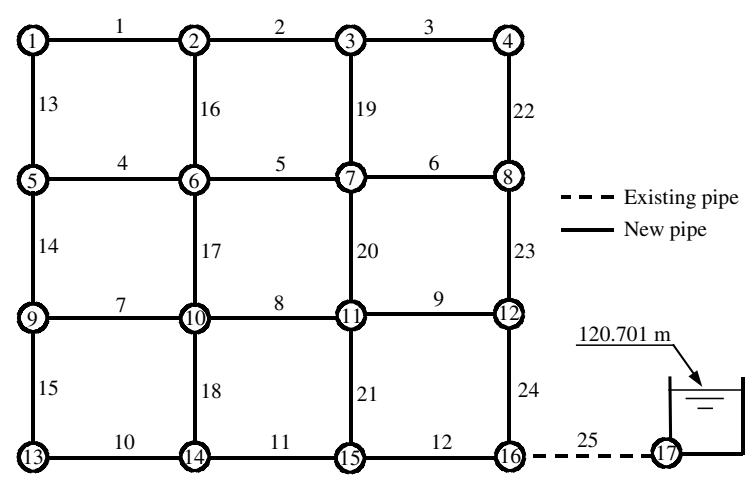

Fig. 4. Network 4 (Gessler, 1981).

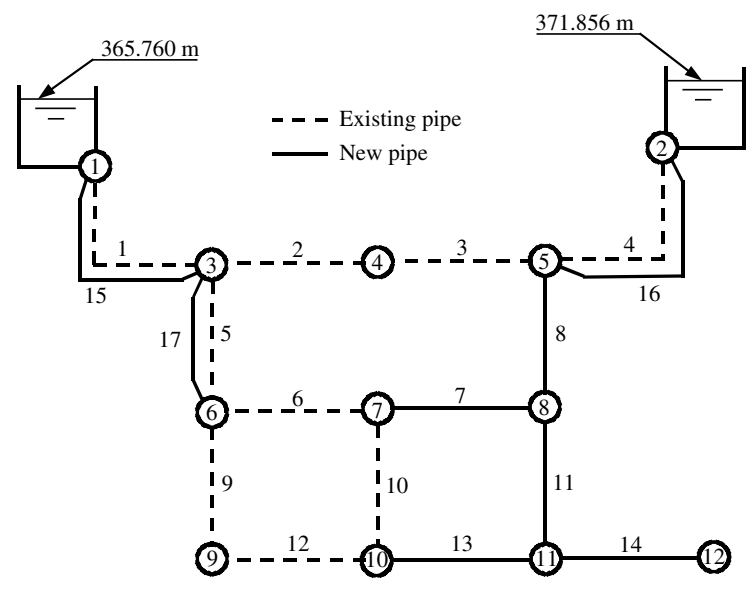

Fig. 5. Network 5 (Walski et al., 1990).

the tabu list size, the aspiration criterion, and the termination criteria.

\subsection{Initial solution}

There are many ways to find a feasible starting solution. This solution must observe the hydraulic equilibrium constraints and the constraints regarding node heads. To establish a general rule easy to implement, a configuration where each pipe has the highest diameter of the commercial set is proposed as starting solution. This starting solution will therefore play the role of the current solution. Then the mechanism for generating neighbor solutions is applied.

\subsection{Neighborhood structures}

Neighborhood structures will vary with algorithm behavior. The move considered first consists of reducing the diameter of one pipe at a time. Therefore neighbor solutions are characterized by configurations in which all pipes but one have the same diameter as in the current configuration. The new current solution will be the one presenting the least-cost while meeting hydraulic constraints. The new current solution is selected by evaluating the cost of each configuration and solving the hydraulic equilibrium equations. For this purpose, a hydraulic simulator is incorporated into the algorithm. The hydraulic model assumes steady state flow conditions and the mass conservation law equations are rewritten in terms of node heads.

The flow is computed by

$Q_{k}=\operatorname{sgn}\left(\Delta H_{k}\right)\left(\frac{\left|\Delta H_{k}\right|}{w\left(\frac{L_{k}}{C_{k}^{\alpha} D_{k}^{\beta}}\right)}\right)^{1 / \alpha}, \quad \forall k \in N P$.

The mass conservation law can consequently be written as follows:

$\sum_{i \in N P_{n}} \operatorname{sgn}\left(\Delta H_{k}\right)\left(\frac{\left|\Delta H_{k}\right|}{w\left(\frac{L_{k}}{C_{k}^{\alpha} D_{k}^{\beta}}\right)}\right)^{1 / \alpha}=S_{n}, \quad \forall n \in N N$.

This yields a set of nonlinear equations that is solved by the method proposed in Gessler (1981). The flow in each pipe and the pressure in each node are obtained, thereby allowing the evaluation of head constraints.

When a local optimum is reached, the move consists of increasing the diameter of one pipe. The new current solution will be defined according to the rules established by the diversification procedure, while meeting the hydraulic constraints.

\subsection{Diversification procedure}

When a local optimal solution is reached, the neighbor solutions are generated by increasing pipe diameters. The information given by a long-term 
memory function will allow the exploration of other areas of the solution space. This information can synthesize many attributes and can be combined in many ways. Matrixes storing the number of times a pipe diameter is modified, or the number of times a pipe has been ascribed a particular diameter size, are examples of the information provided by a long-term memory function that can be used for overcoming local optima. Of the different implementations tried, two worked better than the others:

- the configuration to be chosen is the one in which the pipe whose size was increased presents the lowest value of a parameter given by multiplying the number of changes in the diameter of the pipe during the search procedure by the number of times that the pipe was ascribed the increased diameter size (implementation T.S.1);

- the configuration to be chosen is the one in which the pipe whose size was increased has maintained the same diameter for the longest period in previous iterations (implementation T.S.2).

\subsection{Tabu moves, tabu list size and aspiration criterion}

The move underlying the definition of the new current solution (i.e. the modification of the size of the pipe whose diameter has been changed) becomes a tabu move and enters the tabu list. The tabu tenure parameter undoubtedly influences the final solution. In our implementation, a fixed value and a variable random value were both tried for this parameter. Figs. 6 and 7, and Figs. 8 and 9 show the results of implementations T.S.1 and T.S.2 respectively, where fixed values for a tabu tenure parameter were used (for networks 2 and 3 ). It is clear that when the tabu tenure takes values closer to the number of pipes in each network (network 2 has 34 pipes and network 3 has 21 pipes) the quality of the solution deteriorates. Another aspect to be noted when fixed tabu tenure parameters are used is that the best solutions known for networks $2\left(6.056 \times 10^{6}\right.$ dollars $)$ and network $3\left(37.1 \times 10^{6}\right.$ dollars $)$ are achieved only once: in the T.S.2 implementation with tabu ten-

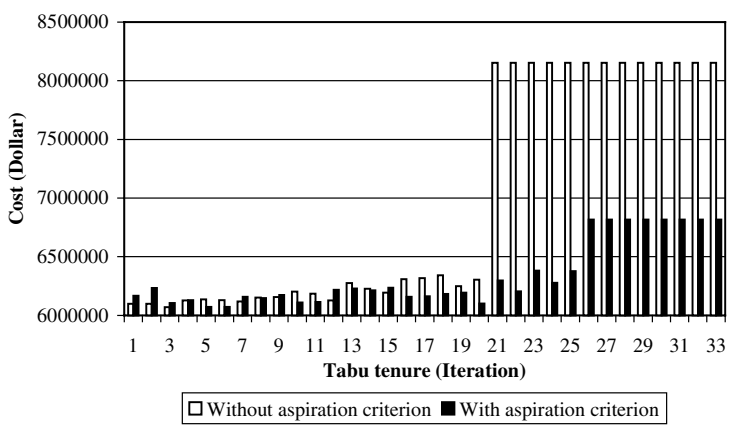

Fig. 6. Network 2-implementation T.S.1.

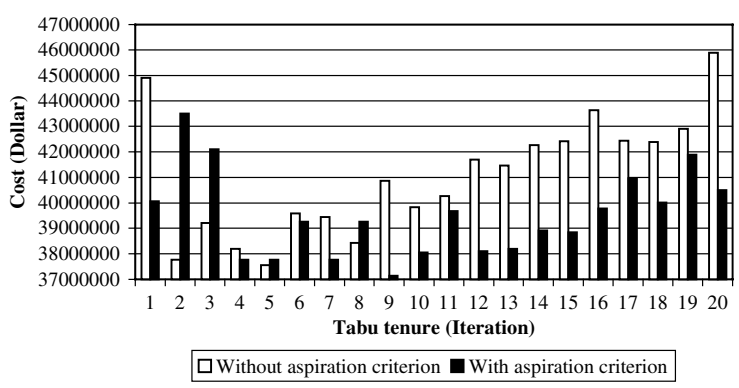

Fig. 7. Network 3-implementation T.S.1.

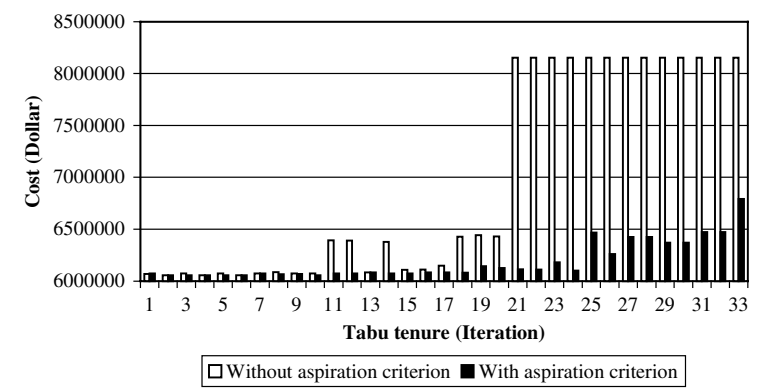

Fig. 8. Network 2-implementation T.S.2.

ure $=2$ or 4 for network 2 ; and in the implementation T.S. 1 with tabu tenure $=9$ for network 3 . But when this parameter is defined randomly an improvement is accomplished. Fig. 10 displays the solutions obtained by T.S.2 implementation for network 2 with a random tabu tenure parameter, using different pseudorandom generator seed numbers. Only in two cases is the best-known historical solution not found (and even in these 


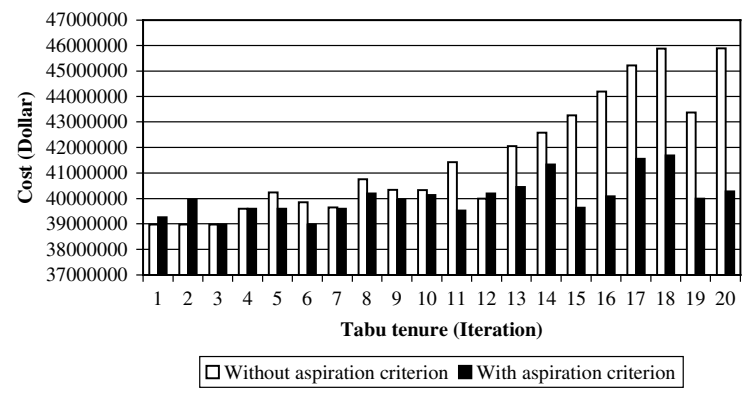

Fig. 9. Network 3-implementation T.S.2.

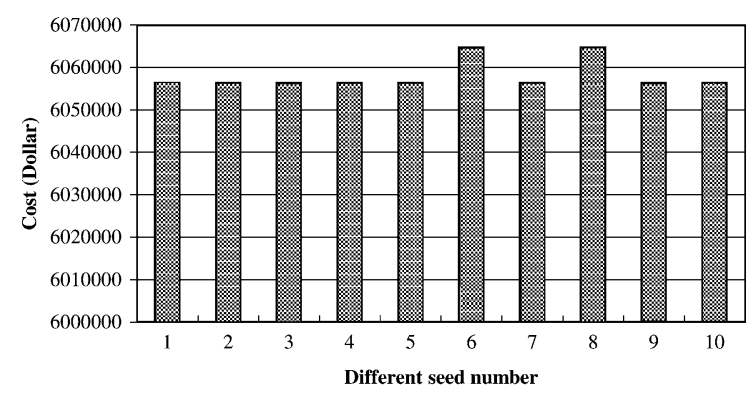

Fig. 10. Network 2-implementation T.S.2 (with random tabu tenure and aspiration criterion).

two cases the difference is very small, being $0.24 \%$ from the best known solution).

An aspiration criterion is activated to overcome the tabu status of a move whenever the solution then produced is better than the best historical solution achieved. This criterion will be effective only after a local optimum is reached.

Figs. 6-9 show the effect of using, or not using, an aspiration criterion. In general, when the aspiration criterion is not used, the best solutions known for networks 2 and 3 are not attained (except for network 2 when the parameter tabu tenure takes values of two or four in the T.S.2 implementation). On the whole, it can be said that the use of an aspiration criterion and a random tabu tenure parameter contribute to an improved final solution.

\subsection{Termination criteria}

Two termination criteria were used: a maximum number of iterations (first criterion), and a maxi- mum number of iterations without improvement of the solution (second criterion). The quality of the final solution and the computer running time are both influenced by the termination criteria. A maximum of 10,000 iterations (first criterion), and a maximum of 3500 for the number of iterations to be performed without solution improvement (second criterion) produced solutions of similar quality, but the implementation of the first criterion is clearly more time consuming.

\subsection{Results}

Table 1 presents different authors' solutions, as reported in the literature as well as T.S.1 and T.S.2 solutions. The costs are expressed in millions of dollars, except those for network 1, which are expressed in monetary units. Three types of solution are reported, according to the methods used. As has been pointed out, split-pipe and continuous diameter designs are not appropriate solutions for this kind of problem. Realistic designs need discrete diameter solutions. The diameters corresponding to networks 1 and 2 are the same as those presented in Cunha and Sousa (1999), and the diameters corresponding to network 3 are the same as those presented in Cunha and Sousa (2001). The diameters for networks 4 and 5 are shown in Tables 2 and 3. Analysis of these tables shows the new combination of pipe sizes, determined by tabu search, that produced better solution costs.

Gessler $(1981,1982)$ used an enumeration procedure to examine all possible combinations. The explosion of the combinatorial dimension was dealt with by grouping together some pipes that were assigned the same diameter. The efficiency of tabu search is enhanced by analyzing each pipe rather than groups of pipes, giving better solutions in comparison to those of Gessler (1981, 1982). This is particularly evident in the results shown in Table 2 for network 4. Various new diameter combinations can be indicated for groups of pipes assigned the same diameter by Gessler (1981). For example, pipes 10,11 , and 12 in network 4 (min. diameter $50.8 \mathrm{~mm}$ ) were assigned the diameter $203.2 \mathrm{~mm}$ in Gessler (1981), and T.S.2 reduces the diameter of pipe 10 to $50.8 \mathrm{~mm}$. New diameter 
Table 1

Solution costs for the five networks

\begin{tabular}{|c|c|c|c|c|c|c|c|}
\hline \multirow[t]{3}{*}{ Authors } & \multicolumn{6}{|c|}{ Networks } & \multirow[t]{3}{*}{ Solution } \\
\hline & \multirow[t]{2}{*}{1} & \multirow[t]{2}{*}{2} & \multirow[t]{2}{*}{3} & \multicolumn{2}{|l|}{4} & \multirow[t]{2}{*}{5} & \\
\hline & & & & $\begin{array}{l}D_{\min }=2 \\
\text { in. }\end{array}$ & $\begin{array}{l}D_{\min }=4 \\
\text { in. }\end{array}$ & & \\
\hline Alperovits and Shamir (1977) & 498 & & & & & & Split-pipe \\
\hline Gessler (1981) & & & & 834 & 858 & & Discrete diameters \\
\hline Gessler (1982) & & & 41.8 & & & & Discrete diameters \\
\hline Morgan and Goulter (1985) & & & 38.9 & & & & Split-pipe \\
\hline Morgan and Goulter (1985) & & & 39.2 & & & & Discrete diameters \\
\hline Goulter et al. (1986) & 435 & & & & & & Split-pipe \\
\hline Kessler (1988) & & & 39.0 & & & & Split-pipe \\
\hline Kessler and Shamir (1989) & 418 & & & & & & Split-pipe \\
\hline Fujiwara and Khang (1990) & & & 36.6 & & & & Split-pipe \\
\hline Fujiwara and Khang $(1990,1991)$ & & 6116 & & & & & Continuous diameters \\
\hline Fujiwara and Khang $(1990,1991)$ & & 6319 & & & & & Split-pipe \\
\hline Walski et al. (1990) & & & & & & 1884.432 & Discrete diameters \\
\hline Sonak and Bhave (1993) & & 6045 & & & & & Split-pipe \\
\hline Murphy et al. (1993) & & & 38.8 & & & & Discrete diameters \\
\hline Eiger et al. (1994) & 402 & 6027 & & & & & Split-pipe \\
\hline Loganathan et al. (1995) & & & 38.0 & & & & Split-pipe \\
\hline Dandy et al. (1996) & & & 38.8 & & & & Discrete diameters \\
\hline Varma et al. (1997) & & 6000 & & & & & Continuous diameters \\
\hline Varma et al. (1997) & & 6162 & & & & & Discrete diameters \\
\hline Savic and Walters (1997) & 419 & 6056 & 37.1 & & & & Discrete diameters \\
\hline Sousa and Cunha (1998) & 419 & 6056 & 37.1 & & & & Discrete diameters \\
\hline Cunha and Sousa (1999) & 419 & 6056 & & & & & Discrete diameters \\
\hline Sousa and Cunha (1999) & & & & 810 & 830 & 1750.320 & Discrete diameters \\
\hline T.S.1 & 420 & 6056 & 37.1 & 810 & 830 & 1727.616 & Discrete diameters \\
\hline T.S.2 & 420 & 6056 & 37.1 & 808 & 830 & 1727.616 & Discrete diameters \\
\hline
\end{tabular}

combinations (e.g. reducing the diameters of pipes 11 and 13) produce a better solution for network 5 vis-à-vis the solution provided by Walski et al. (1990). The results also show that tabu search algorithms achieve an improvement for some of the networks (networks 4 and 5) vis-à-vis the solutions provided by simulated annealing (Sousa and Cunha, 1999).

Computer running times using a Pentium PC at $433 \mathrm{MHz}$ are displayed in Table 4. It must be emphasized that the solution space, where a configuration that produces the least-cost for each network is to be found, is very large. Taking network 2 , for example, which has 34 pipes, and using a commercial diameter set containing six different diameters, the solution space contains $6^{34}=$ $2.87 \times 10^{26}$ different possible network designs. The search procedure can thus be considered quite efficient.

As mentioned in the introduction, since a global optimal solution is not known for these problems, it is very important to evaluate different heuristic methods to find the most suitable. Our aim, in this paper, has been to tailor a tabu search algorithm to achieve high quality least-cost solutions for looped water distribution networks. This objective was attained, but when the results provided by the three modern heuristic methods are analyzed, it is still hard to arrive at a general conclusion about the most appropriate heuristic for dealing with the problem defined here.

The ability of the tabu search algorithm that has been built to overcome local optima solutions should be indicated. To test it, some local optima 
Table 2

Solution for network 4

\begin{tabular}{|c|c|c|c|c|c|c|c|c|}
\hline \multirow{3}{*}{$\begin{array}{l}\text { Pipe } \\
\text { number }\end{array}$} & \multirow[t]{3}{*}{ Length $(\mathrm{m})$} & \multirow{3}{*}{$\begin{array}{l}\text { Hazen- } \\
\text { Williams } \\
\text { coefficients }\end{array}$} & \multicolumn{6}{|c|}{ Diameter (mm) } \\
\hline & & & \multicolumn{3}{|c|}{ Minimum diameter 2 in. $=50.8 \mathrm{~mm}$} & \multicolumn{3}{|c|}{ Minimum diameter 4 in. $=101.6 \mathrm{~mm}$} \\
\hline & & & $\begin{array}{l}\text { Gessler } \\
(1981)\end{array}$ & $\begin{array}{l}\text { Sousa and } \\
\text { Cunha } \\
(1999)\end{array}$ & T.S.2 & $\begin{array}{l}\text { Gessler } \\
(1981)\end{array}$ & $\begin{array}{l}\text { Sousa and } \\
\text { Cunha } \\
\text { (1999) }\end{array}$ & $\begin{array}{l}\text { T.S. } 1 \text { and } \\
\text { T.S. } 2\end{array}$ \\
\hline 1 & 365.76 & 120 & 152.4 & 50.8 & 50.8 & 101.6 & 101.6 & 101.6 \\
\hline 2 & 365.76 & 120 & 152.4 & 50.8 & 50.8 & 101.6 & 101.6 & 101.6 \\
\hline 3 & 365.76 & 120 & 152.4 & 101.6 & 50.8 & 101.6 & 101.6 & 101.6 \\
\hline 4 & 365.76 & 120 & 50.8 & 203.2 & 203.2 & 101.6 & 101.6 & 101.6 \\
\hline 5 & 365.76 & 120 & 50.8 & 50.8 & 50.8 & 101.6 & 101.6 & 101.6 \\
\hline 6 & 365.76 & 120 & 50.8 & 50.8 & 101.6 & 101.6 & 101.6 & 101.6 \\
\hline 7 & 365.76 & 120 & 50.8 & 50.8 & 50.8 & 101.6 & 152.4 & 152.4 \\
\hline 8 & 365.76 & 120 & 50.8 & 203.2 & 101.6 & 101.6 & 152.4 & 152.4 \\
\hline 9 & 365.76 & 120 & 50.8 & 203.2 & 50.8 & 101.6 & 101.6 & 101.6 \\
\hline 10 & 365.76 & 120 & 203.2 & 50.8 & 50.8 & 203.2 & 101.6 & 101.6 \\
\hline 11 & 365.76 & 120 & 203.2 & 101.6 & 203.2 & 203.2 & 152.4 & 152.4 \\
\hline 12 & 365.76 & 120 & 203.2 & 101.6 & 203.2 & 203.2 & 203.2 & 203.2 \\
\hline 13 & 243.84 & 120 & 152.4 & 203.2 & 203.2 & 152.4 & 203.2 & 203.2 \\
\hline 14 & 243.84 & 120 & 152.4 & 50.8 & 50.8 & 152.4 & 152.4 & 152.4 \\
\hline 15 & 243.84 & 120 & 152.4 & 50.8 & 50.8 & 152.4 & 101.6 & 101.6 \\
\hline 16 & 243.84 & 120 & 50.8 & 50.8 & 50.8 & 101.6 & 101.6 & 101.6 \\
\hline 17 & 243.84 & 120 & 50.8 & 203.2 & 203.2 & 101.6 & 101.6 & 101.6 \\
\hline 18 & 243.84 & 120 & 50.8 & 50.8 & 203.2 & 101.6 & 101.6 & 101.6 \\
\hline 19 & 243.84 & 120 & 50.8 & 50.8 & 101.6 & 101.6 & 101.6 & 101.6 \\
\hline 20 & 243.84 & 120 & 50.8 & 50.8 & 50.8 & 101.6 & 101.6 & 101.6 \\
\hline 21 & 243.84 & 120 & 50.8 & 101.6 & 101.6 & 101.6 & 152.4 & 152.4 \\
\hline 22 & 243.84 & 120 & 152.4 & 101.6 & 50.8 & 152.4 & 101.6 & 101.6 \\
\hline 23 & 243.84 & 120 & 152.4 & 101.6 & 101.6 & 152.4 & 101.6 & 101.6 \\
\hline 24 & 243.84 & 120 & 152.4 & 203.2 & 101.6 & 152.4 & 152.4 & 152.4 \\
\hline 25 & 914.40 & 120 & 254.0 & 254.0 & 254.0 & 254.0 & 254.0 & 254.0 \\
\hline \multicolumn{3}{|c|}{ Solution cost (\$) } & 834,000 & 810,000 & 807,999 & 858,000 & 830,000 & 830,000 \\
\hline
\end{tabular}

solutions regarding New York network (network 3 ) included in the literature were used as starting solutions to run the tabu search algorithm developed. They are presented in Table 5 for Morgan and Goulter (1985) and Murphy et al. (1993) and in Table 6 for Gessler (1982). This author did not use the same commercial diameter set as is usually found in the literature. For the sake of comparison, the diameters used in the Gessler (1982) solution were converted into the nearest ones included in those sets.

Fig. 11 shows how the algorithm develops when it starts with the Gessler (1982) solution. A zoom in this figure, taking iterations from 0 to 160 (Fig.
12), shows that the optimal solution (37.1 M\$ dollars) is obtained in iteration 124. Figs. 13 and 14 display the behavior of the algorithm when the Morgan and Goulter (1985) and Murphy et al. (1993) solutions are used as starting solutions (the optimal solution is obtained in iteration 103 for the first case and in iteration 300 for the second case).

In Figs. 12 and 13 it can be seen that the diversification procedure takes place very early (in the fifth iteration and in the fourth iteration when Gessler (1982) and Morgan and Goulter (1985) are the respective starting solutions). When the Murphy et al. (1993) solution is used as 
Table 3

Solution for network 5

\begin{tabular}{lcclrl}
\hline Pipe number & Length $(\mathrm{m})$ & $\begin{array}{l}\text { Hazen-Williams } \\
\text { coefficient }\end{array}$ & Diameter $(\mathrm{mm})$ & \\
\cline { 5 - 6 } & & & Walski et al. $(1990)$ & Sousa and Cunha (1999) & T.S.1 and T.S.2 \\
\hline 1 & 4828.032 & 75 & 355.6 & 355.6 & 355.6 \\
2 & 1609.344 & 80 & 254.0 & 254.0 & 254.0 \\
3 & 1609.344 & 80 & 254.0 & 254.0 & 254.0 \\
4 & 6437.376 & 80 & 254.0 & 254.0 & 254.0 \\
5 & 1609.344 & 80 & 254.0 & 254.0 & 254.0 \\
6 & 1609.344 & 100 & 203.2 & 203.2 & 203.2 \\
7 & 1609.344 & 120 & 203.2 & 203.2 & 203.2 \\
8 & 1609.344 & 120 & 304.8 & 304.8 & 304.8 \\
9 & 1609.344 & 80 & 254.0 & 254.0 & 254.0 \\
10 & 1609.344 & 100 & 101.6 & 101.6 & 101.6 \\
11 & 1609.344 & 120 & 304.8 & 203.2 & 254.0 \\
12 & 1609.344 & 100 & 203.2 & 203.2 & 203.2 \\
13 & 1609.344 & 120 & 203.2 & 152.4 & 101.6 \\
14 & 1609.344 & 120 & 203.2 & 254.0 & 203.2 \\
15 & 4828.032 & 120 & 0.0 & 0.0 & 0.0 \\
16 & 6437.376 & 120 & 355.6 & 355.6 & 355.6 \\
17 & 1609.344 & 120 & 0.0 & 0.0 & 0.0 \\
\hline
\end{tabular}

Table 4

Running times (in seconds)

\begin{tabular}{lllllll}
\hline & Networks & & & \\
\cline { 2 - 7 } & 1 & 2 & 3 & $4\left(D_{\min }=2\right.$ in. $)$ & $4\left(D_{\min }=4\right.$ in. $)$ & 5 \\
\hline T.S.1 & 16 & 391 & 54 & 71 & 46 & 46 \\
T.S.2 & 16 & 445 & 60 & 94 & 65 & 55 \\
\hline
\end{tabular}

starting solution, the diversification procedure occurs in the first iteration. It should be noted that when the starting solution is that described in Section 4.1, i.e. a configuration where each pipe has the highest diameter of the commercial set, the diversification procedure takes place in iteration 144.

\section{Conclusions}

A tabu search algorithm has been constructed to enable the least-cost design of looped water distribution networks to be found. The fine-tuning of its parameters permits high quality solutions to be achieved, and the results obtained reveal the ability of the algorithm to cope with the combi- natorial characteristics of this problem. Some improvements in the results achieved with tabu search algorithms can be indicated.

The small number of case studies available in the literature means that the comparison of the solutions provided by the three modern heuristic techniques (genetic algorithms, simulated annealing and tabu search) does not allow general conclusions to be drawn about the heuristic most suitable for solving the problem proposed here. Since the solution of realistic network problems would benefit greatly from the application of heuristics, the authors suggest that a database of networks should be set up. This would enable the conclusions drawn from studies carried out on these networks to be more easily compared by researchers in this field. 
Table 5

Morgan and Goulter (1985) and Murphy et al. (1993) solutions

\begin{tabular}{|c|c|c|c|c|c|}
\hline \multirow[t]{2}{*}{ Pipe } & \multirow[t]{2}{*}{$L(\mathrm{~m})$} & \multicolumn{2}{|c|}{ Morgan and Goulter (1985) } & \multicolumn{2}{|c|}{ Murphy et al. (1993) } \\
\hline & & $\overline{D \text { (in.) }}$ & $D(\mathrm{~mm})$ & $\overline{D \text { (in.) }}$ & $D(\mathrm{~mm})$ \\
\hline 1 & 3535.723 & 0 & 0 & 0 & 0 \\
\hline 2 & 6035.113 & 0 & 0 & 0 & 0 \\
\hline 3 & 2225.067 & 0 & 0 & 0 & 0 \\
\hline 4 & 2529.871 & 0 & 0 & 0 & 0 \\
\hline 5 & 2621.312 & 0 & 0 & 0 & 0 \\
\hline 6 & 5821.751 & 0 & 0 & 0 & 0 \\
\hline 7 & 2926.116 & 144 & 3657.6 & 0 & 0 \\
\hline 8 & 3810.046 & 0 & 0 & 0 & 0 \\
\hline 9 & 2926.116 & 0 & 0 & 0 & 0 \\
\hline 10 & 3413.802 & 0 & 0 & 0 & 0 \\
\hline 11 & 4419.654 & 0 & 0 & 0 & 0 \\
\hline 12 & 3718.605 & 0 & 0 & 0 & 0 \\
\hline 13 & 7345.769 & 0 & 0 & 0 & 0 \\
\hline 14 & 6431.358 & 0 & 0 & 0 & 0 \\
\hline 15 & 4724.457 & 0 & 0 & 120 & 3048 \\
\hline 16 & 8046.818 & 96 & 2438.4 & 84 & 2133.6 \\
\hline 17 & 9509.876 & 96 & 2438.4 & 96 & 2438.4 \\
\hline 18 & 7315.289 & 84 & 2133.6 & 84 & 2133.6 \\
\hline 19 & 4389.173 & 60 & 1524 & 72 & 1828.8 \\
\hline 20 & 11704.462 & 0 & 0 & 0 & 0 \\
\hline 21 & 8046.818 & 84 & 2133.6 & 72 & 1828.8 \\
\hline \multicolumn{2}{|c|}{ Cost (\$ million) } & \multicolumn{2}{|c|}{39.2} & \multicolumn{2}{|c|}{38.8} \\
\hline
\end{tabular}

Table 6

Gessler (1982) solution

\begin{tabular}{|c|c|c|c|}
\hline \multirow[t]{2}{*}{ Pipe } & \multicolumn{2}{|c|}{ Gessler (1982) } & \multirow{2}{*}{$\begin{array}{l}D \text { selected } \\
(\mathrm{mm})\end{array}$} \\
\hline & $D$ (in.) & $D(\mathrm{~mm})$ & \\
\hline 1 & 0 & 0 & 0 \\
\hline 2 & 0 & 0 & 0 \\
\hline 3 & 0 & 0 & 0 \\
\hline 4 & 0 & 0 & 0 \\
\hline 5 & 0 & 0 & 0 \\
\hline 6 & 0 & 0 & 0 \\
\hline 7 & 100 & 2540 & 2438.4 \\
\hline 8 & 100 & 2540 & 2438.4 \\
\hline 9 & 0 & 0 & 0 \\
\hline 10 & 0 & 0 & 0 \\
\hline 11 & 0 & 0 & 0 \\
\hline 12 & 0 & 0 & 0 \\
\hline 13 & 0 & 0 & 0 \\
\hline 14 & 0 & 0 & 0 \\
\hline 15 & 0 & 0 & 0 \\
\hline 16 & 100 & 2540 & 2438.4 \\
\hline 17 & 100 & 2540 & 2438.4 \\
\hline 18 & 80 & 2032 & 2133.6 \\
\hline 19 & 60 & 1524 & 1524 \\
\hline 20 & 0 & 0 & 0 \\
\hline 21 & 80 & 2032 & 2133.6 \\
\hline Cost (\$ million) & & .8 & \\
\hline
\end{tabular}

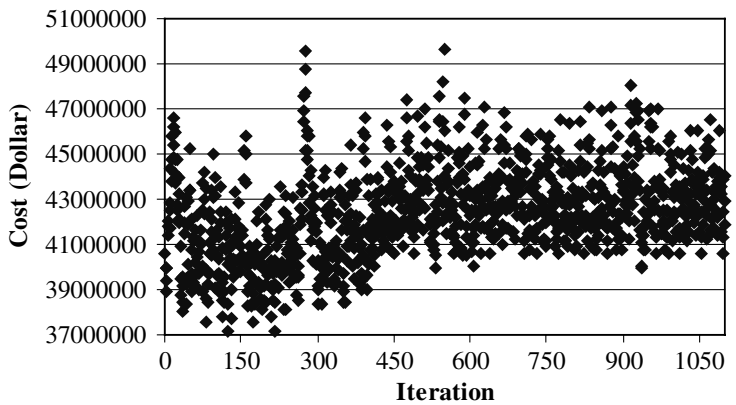

Fig. 11. Algorithm development when the Gessler (1982) solution is the starting solution.

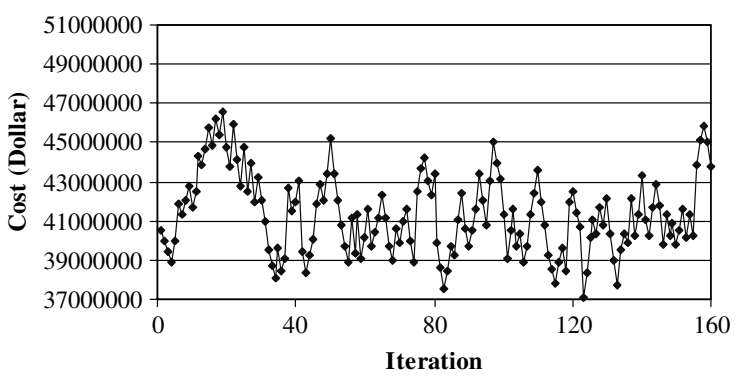

Fig. 12. Initial iterations when the Gessler (1982) solution is the starting solution. 


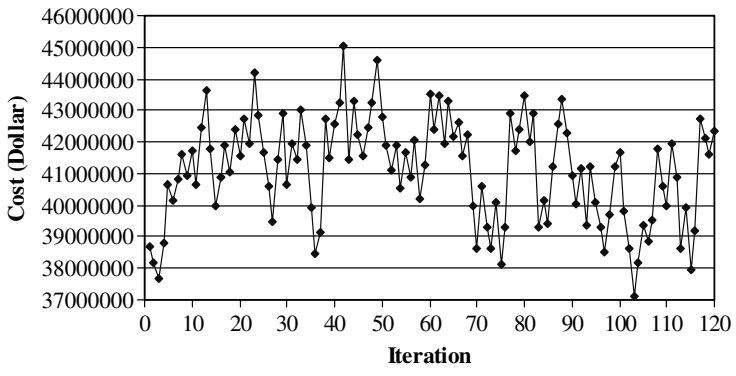

Fig. 13. Initial iterations when the Morgan and Goulter (1985) solution is the starting solution.

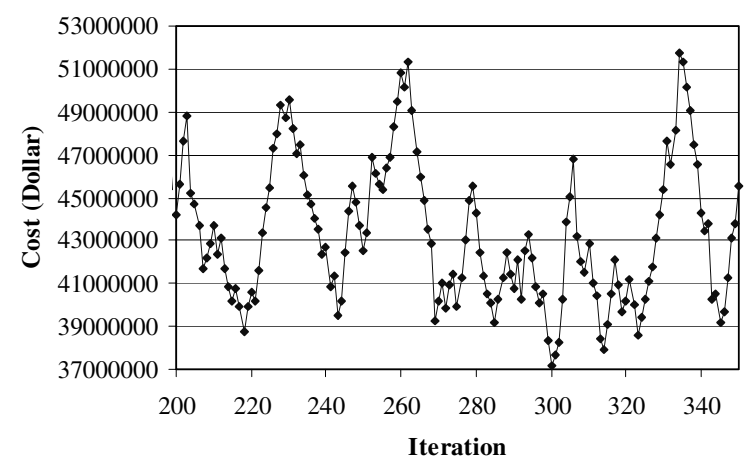

Fig. 14. Initial iterations when the Murphy et al. (1993) is the starting solution.

The results obtained with tabu search algorithms seem very promising, encouraging us to go further in future works by including pumps and other devices, reservoir sizing, etc.

\section{References}

Alperovits, E., Shamir, U., 1977. Design of optimal water distribution systems. Water Resources Research 13 (6), 885900.

Cunha, M.C., Sousa, J., 1999. Water distribution network design optimization: Simulated annealing approach. Journal of Water Resources Planning and Management, ASCE 125 (4), 215-221.

Cunha, M.C., Sousa, J., 2001. Hydraulic infrastructures design using simulated annealing. Journal of Infrastructure Systems, ASCE 7 (1), 32-39.

Dandy, G.C., Simpson, A.R., Murphy, L.J., 1996. An improved genetic algorithm for pipe network optimization. Water Resources Research 32 (2), 449-458.

Eiger, G., Shamir, U., Ben-Tal, A., 1994. Optimal design of water distribution networks. Water Resources Research 30 (9), 2637-2646.
Faigle, U., Kern, W., 1992. Some convergence results for probabilistic tabu search. ORSA Journal on Computing 4 (1), 32-37.

Fujiwara, O., Khang, D.B., 1990. A two-phase decomposition method for optimal design of looped water distribution networks. Water Resources Research 26 (4), 539-549.

Fujiwara, O., Khang, D.B., 1991. Correction to a two-phase decomposition method for optimal design of looped water distribution networks. Water Resources Research 27 (5), 985-986.

Fujiwara, O., Jenchaimahakoon, B., Edirisinghe, N.C.P., 1987. A modified linear programming gradient method for optimal design of looped water distribution networks. Water Resources Research 23 (6), 977-982.

Gessler, J., 1981. Analysis of pipe networks. In: Chaudhry, M.H., Yevjevich, V. (Eds.), Closed-conduit Flow. Water Resources Publications, pp. 61-69.

Gessler, J., 1982. Optimization of pipe networks. In: Proc. Inst. on Urban Hydr. and Sediment Control, University of Kentucky, Lexington, KY, pp. 165-171.

Gessler, J., 1985. Pipe network optimization by enumeration. In: Torno, H. (Ed.), Computer Applications in Water Resources. ASCE Water Resources Planning and Management Division Conference, Buffalo, NY, p. 572.

Glover, F., Laguna, M., 1997. Tabu Search. Kluwer Academic Publishers, Dordrecht.

Goldberg, D.E., Kuo, C.H., 1987. Genetic algorithms in pipeline optimization. Journal of Computing in Civil Engineering 1 (2), 128-141.

Goulter, I.C., Lussier, B.M., Morgan, D.R., 1986. Implications of head loss path choice in the optimization of water distribution networks. Water Resources Research 22 (5), 819-822.

Hadji, G., Murphy, L.J., 1990. Genetic algorithms for pipe network optimization. 4th Year Student Civil Engineering Research Report, University of Adelaide, Australia.

Kessler, A., 1988. Optimal design of water supply networks using graph theory. D.Sc. thesis, Technion-Israel Inst. Technol. Haifa, Israel.

Kessler, A., Shamir, U., 1989. Analysis of the linear programming gradient method for optimal design of water supply networks. Water Resources Research 25 (7), 1469-1480.

Lansey, K.E., Mays, L.W., 1989. Optimization model for design of water distribution systems. In: Mays, L.R. (Ed.), Reliability Analysis of Water Distribution Systems. ASCE, New York, NY.

Loganathan, G.V., Greene, J.J., Ahn, T.J., 1995. Design heuristic for globally minimum cost water-distribution systems. Journal of Water Resources Planning and Management, ASCE 121 (2), 182-192.

Morgan, D.R., Goulter, I.C., 1985. Optimal urban water distribution design. Water Resources Research 21 (5), 642-652.

Murphy, L.J., Simpson, A.R., 1992. Genetic algorithms in pipe network optimization. Research Report No. R93, Dept. of Civ. and Envir. Engrg., Univ. of Adelaide, Australia.

Murphy, L.J., Simpson, A.R., Dandy, G.C., 1993. Design of a network using genetic algorithms. Water 20, 40-42. 
Quindry, G.E., Brill, E.D., Liebman, J.C., 1981. Optimization of looped water distribution systems. Journal of Environmental Engineering, ASCE 107 (4), 665-679.

Savic, D.A., Walters, G.A., 1997. Genetic algorithms for leastcost design of water distribution networks. Journal of Water Resources Planning and Management, ASCE 123 (2), 6777.

Simpson, A.R., Dandy, G.C., Murphy, L.J., 1994. Genetic algorithms compared to other techniques for pipe optimization. Journal of Water Resources Planning and Management, ASCE 120 (4), 423-443.

Sonak, V.V., Bhave, P.R., 1993. Global optimum tree solution for single-source looped water distribution networks subjected to a single loading pattern. Water Resources Research 29 (7), 2437-2443.

Sousa, J., Cunha, M.C., 1998. On solving water distribution network design problems with stochastic optimization techniques. In: Blain, W.R. (Ed.), Hydraulic Engineering Software VII. Computational Mechanics Publications, WIT Press, pp. 141-150.

Sousa, J., Cunha, M.C., 1999. On the quality of a simulated annealing algorithm for water network optimization prob- lems. In: Savic, D., Walters, G. (Eds.), Water Industry Systems: Modelling and Optimization Applications, vol. 2. Research Studies Press Ltd., pp. 333-345.

Varma, K.V.K., Narasimhan, S., Bhallamudi, S.M., 1997. Optimal design of water distribution systems using an NLP method. Journal of Environmental Engineering, ASCE 123 (4), 381-388.

Walski, T.M., 1985. State-of-the-art: Pipe network optimization. Computer Applications in Water Resources, Buffalo, NY.

Walski, T.M., Sjostrom, J.W., Gessler, J., 1990. Water distribution systems: Simulation and sizing. Lewis Publishers, Inc.

Walters, G.A., Cembrowicz, R.G., 1993. Optimal design of water distribution networks. In: Cabrera, E., Martinez, F. (Eds.), Water Supply Systems, State of the Art and Future Trends. Computational Mechanics Publications, Southampton, pp. 91-117.

Walters, G.A., Halhal, D., Savic, D., Quazar, D., 1999. Improved design of "Anytown" distribution network using structured messy genetic algorithm. Urban Water 1 (1), 2328. 